\title{
Application of the AMLprofiler Diagnostic Microarray in the South African Setting
}

\author{
S. S. Kappala, ${ }^{1}$ M. Alessandrini, ${ }^{1}$ T. Matlhako, ${ }^{2}$ E. Beltchev, ${ }^{2}$ R. Pool, ${ }^{2}$ and M. S. Pepper ${ }^{1}$ \\ ${ }^{1}$ Institute for Cellular and Molecular Medicine, Department of Immunology, Faculty of Health Sciences, \\ and SAMRC Extramural Unit for Stem Cell Research and Therapy, University of Pretoria, Pretoria, South Africa \\ ${ }^{2}$ Department of Haematology, Faculty of Health Sciences, University of Pretoria and National Health Laboratory Services, \\ Pretoria, South Africa
}

Correspondence should be addressed to M. S. Pepper; michael.pepper@up.ac.za

Received 21 May 2017; Revised 9 August 2017; Accepted 12 October 2017; Published 7 November 2017

Academic Editor: Linda Sommese

Copyright (c) 2017 S. S. Kappala et al. This is an open access article distributed under the Creative Commons Attribution License, which permits unrestricted use, distribution, and reproduction in any medium, provided the original work is properly cited.

Acute myeloid leukemia (AML) is characterized by proliferation of the myeloid lineage and accumulation of immature hematopoietic cells in the bone marrow and is typified by marked heterogeneity both in response to treatment and survival. AMLprofiler is a qualitative in vitro diagnostic microarray incorporating seven molecular biomarkers used to diagnose and predict posttherapy survival rates. In this study, we compared AMLprofiler to routine AML diagnostic methodologies employed in South Africa, focusing on consistency of the results, cost, and time to result. RNA was isolated from bone marrow and peripheral blood samples from patients with de novo AML and was processed using Affymetrix Gene Profiling Reagent kits. The results from AMLprofiler and standard methodologies were highly comparable. In addition, many samples were determined to be positive for biomarkers not routinely investigated in South Africa, namely, CEBPA double mutants, NPM1 variants, and altered expression levels of BAALC and EVI1. 38\% of samples presented with no positive biomarker; AMLprofiler nonetheless enabled $26 \%$ of AML patients to be classified into either favorable or poor prognostic categories. This study highlights the comprehensive nature of the microarray. Decreased time to result and refinement of risk stratification are notable benefits.

\section{Introduction}

Acute myeloid leukemia (AML) is a heterogeneous disorder both biologically and clinically, characterized by accumulation of immature hematopoietic cells in the bone marrow [1]. These cells are commonly referred to as leukemic blasts and lack the capacity to self-renew. Clinical symptoms include fever, fatigue, and spontaneous mucosal and cutaneous bleeding. The leading cause of death is bone marrow failure that results in anaemia, neutropenia, and thrombocytopenia [2]. Diagnosis of AML involves a combination of morphological, cytochemical, and immunophenotyping techniques. Conventional cytogenetic analysis constitutes a crucial part of the standard diagnostic workup for AML patients, as about 55\% have chromosomal abnormalities [3].

The World Health Organization (WHO) classifies AML into four major groups based on clinical, morphological, immunophenotypic, and genetic features: (1) AML with recurrent chromosomal abnormalities; (2) therapy-related AML; (3) myelodysplastic syndrome- (MDS-) associated AML; and (4) AML not otherwise specified (NOS) [4]. Prognostic classification is based on data derived from cytogenetics, fluorescent in situ hybridization (FISH), and molecular biology techniques. The karyotype of AML patients is by far the most important prognostic parameter considered when deciding on a specific treatment regime [5]. The prognostic classification of AML patients includes three categories, namely, favorable, intermediate, and poor risk. Approximately $45 \%$ of AML patients, who are cytogenetically normal (CN-AML), fall into the intermediate prognostic risk category and are often the most challenging to treat. Prognostic classification is a crucial step in the diagnosis of AML, particularly with respect to identifying those at high risk of relapse and also for category-specific treatment options. 
In recent years, the laboratory diagnosis of AML has improved significantly. Microarray technologies, both DNA and gene expression based, are being utilized increasingly for the detection of mutations and changes in gene expression profiles. Additionally, next-generation sequencing technologies are constantly being improved and are also being applied more frequently in this context [6]. Yet, cytogenetic karotyping still remains the gold standard in routine diagnostic procedures for AML patients, which is time-consuming and labor-intensive. Current modalities for AML diagnosis include cytogenetics and molecular approaches (gene mutations and levels of gene expression) which encompasses a wide range of tests when performed individually. The AMLprofiler (SkylineDx, Rotterdam, The Netherlands) is a qualitative in vitro diagnostic microarray that uses RNA chemistry to identify seven key molecular biomarkers used for the diagnosis and prognosis of AML: $\operatorname{inv}(16) / \mathrm{t}(16 ; 16), \mathrm{t}(8 ; 21)$, and $\mathrm{t}(15 ; 17)$; biallelic CEBPA mutation (CEBPAdm); ABD-type NPM1 mutations; $B A A L C$-low and EVI1-high mRNA expression levels. Each of these biomarkers is associated with either a favorable or poor prognosis (Table 1) and has relevance in cases where the samples have also been shown to be devoid of chromosomal aberrations, that is, fall into the category of cytogenetically normal AML (CN-AML).

The AMLprofiler assay thus allows for the standardized assessment of chromosomal aberrations and molecular abnormalities, which are of clinical significance in AML (Table 1). AMLprofiler is aligned with the global best practice for diagnositic tests in AML and encompasses seven molecular variables including gene mutations and gene rearrangements [7]. High levels of expression of several genes including EVI1, BAALC, MN1, and ERG have been found to be prognostically relevant in AML $[8,9]$. In 2013, Brand et al. successfully standardized and validated BAALC and EVI1 gene expression markers in a cohort of intermediate cytogenetic risk AML patients [10]. Low BAALC expression was associated with a favorable prognosis and high expression of EVI1 with an unfavorable prognosis. These genes have been incorporated into the AMLprofiler as independent prognostic factors.

In 2016, Nomdedéu et al. reported findings from a feasibility study on AMLprofiler for patient risk stratification in a multicentre trial, which also included a preliminary comparison with the conventional approach [11]. They analysed both the cost factor and turnaround time of AMLprofiler and compared the results with those obtained using conventional diagnostic methods. They further compared the standard prognostic stratification versus the AMLprofiler and concluded that both methods provided significant clinical information. Their results indicated that AMLprofiler was no more expensive than a conventional molecular approach and turnaround times were similar for both approaches. Therefore, they concluded that AMLprofiler could be successfully applied for AML diagnosis in Spain, in order to rapidly identify AML patients with a good prognosis.

In this study, we set out to evaluate the feasibility of utilizing the AMLprofiler in the South African context and also to assess the possible added prognostic value relative to
TABLE 1: Chromosomal aberrations and molecular marker variants detected by AMLprofiler.

\begin{tabular}{lcc}
\hline Markers on AMLprofiler & $\begin{array}{c}\text { Prognostic } \\
\text { category }\end{array}$ \\
\hline \multirow{2}{*}{ Chromosomal } & $\operatorname{inv}(16)(\mathrm{p} 13 \mathrm{q} 22) /$ & Favorable \\
aberrations & $\mathrm{t}(16 ; 16)(\mathrm{p} 13 ; \mathrm{q} 22)$ & Favorable \\
& $\mathrm{t}(8 ; 21)(\mathrm{q} 22 ; \mathrm{q} 22)$ & Favorable \\
\hline Gene mutations & $\mathrm{t}(15 ; 17)(\mathrm{q} 24 ; \mathrm{q} 21)$ & Favorable \\
& NPBPA double mutant & Favorable \\
\hline \multirow{2}{*}{ Gene expression } & $E V I 1$-high expression & Unfavorable \\
& $B A A L C$-low expression & Favorable \\
\hline
\end{tabular}

standard procedures. We thus aimed to assess the extent of concordance between the results obtained with traditional diagnostic modalities versus AMLprofiler and also determined whether there might be disparities between the AMLprofiler and standard prognostic stratification that include traditional cytogenetic and molecular methods. We also looked at the cost factor and turnaround time required to execute the AMLprofiler test in the current diagnostic setup in South Africa.

\section{Methods}

2.1. Patient Recruitment and Sample Collection. Approval for this study was obtained from the Research Ethics Committee of the Faculty of Health Sciences, University of Pretoria (Ref. number 42/2012). AML patients were recruited via the National Health Laboratory Service (NHLS) at the Universities of Pretoria and the Witwatersrand and from private pathology groups including Ampath Laboratories and Vermaak and Partners Pathologists. Only adult patients diagnosed with de novo AML, based on a blast count of $>20 \%$, were included.

2.2. RNA Isolation. The samples were received at the Institute for Cellular and Molecular Medicine (ICMM) laboratory at the University of Pretoria, where RNA was isolated within 48 hours of sample collection. Mononuclear cells were first separated using Ficoll histopaque (Sigma-Aldrich), after which RNA was isolated using the Qiagen RNAeasy kit according to the manufacturer's protocol. The quantity of RNA was checked using a Nanodrop spectrophotometer and RNA integrity analysed on Agilent's TapeStation 2200 before continuing further with the assay. Analysis of $16 \mathrm{~S}$ and 28S RNA peaks was checked using an RNA Integrity Number (RIN) value. Only samples with a RIN value above 7 were selected for the AMLprofiler procedure.

2.3. AMLprofiler Assay. The AMLprofiler assay includes different experimental steps that follow in a sequential order and that span over a period of 3 days (Figure 1). This assay was performed according to the manufacturer's protocol. Briefly, the initial step involves synthesis of cDNA from RNA in a two-step reaction that includes first strand and 


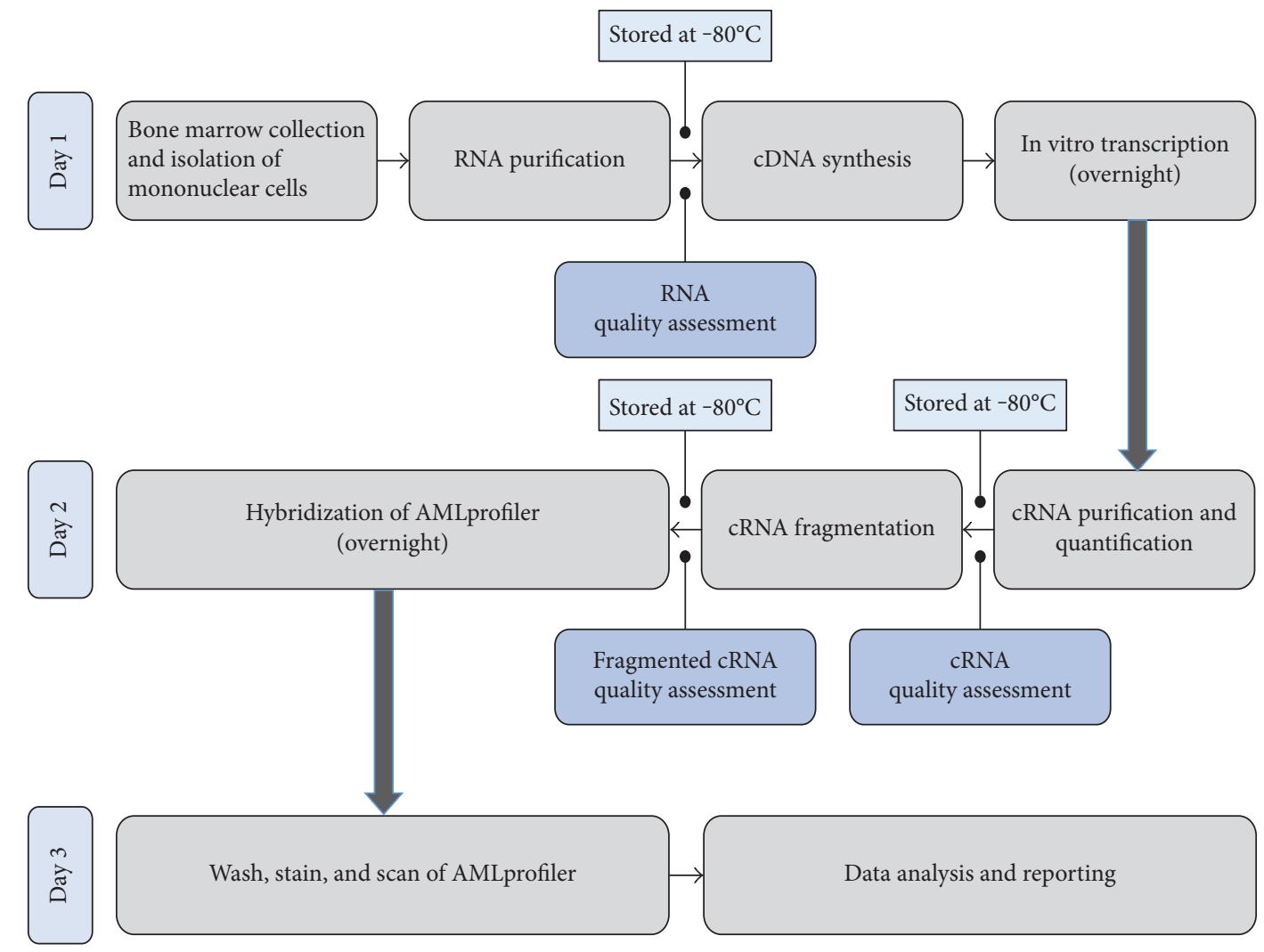

Figure 1: An overview of the AMLprofiler assay. The day to day process flow involved in the AMLprofiler microarray analysis with specific stop points and storage conditions.

second strand synthesis. The third reaction is an in vitro transcription reaction where biotinylated complimentary RNA (cRNA) is synthesized. The cRNA is then purified using magnetic bead separation and analysed using the Agilent TapeStation 2200 to assess for quality and integrity before proceeding to a fragmentation reaction. The cRNA is then fragmented, and the quality was checked again. The final step of the process is the hybridization reaction, where the fragmented cRNA is loaded onto the AMLprofiler microarray and hybridized for 16-17 hours.

2.4. Data Analysis and Reporting. Following overnight hybridization, the AMLprofiler microarray was washed, stained, and scanned. After scanning, the data was transferred through a secured centralized server to SkylineDx, The Netherlands, for analysis, and a report was received in the ICMM laboratory within 15-20 minutes.

The results for chromosomal aberrations and gene mutation/expression markers on the AMLprofiler report are displayed as detected/not detected/invalid assay/not applicable. The result shows "detected" in cases where the marker is present, "not detected" in cases where the marker is absent, and "invalid assay" in cases where the data analysis QC parameters do not meet the acceptance criteria. When a patient is detected to be positive for one of the three chromosomal aberrations-inv(16)(p13q22)/ $\mathrm{t}(16 ; 16)(\mathrm{p} 13 ; \mathrm{q} 22), \mathrm{t}(8 ; 21)(\mathrm{q} 22 ; \mathrm{q} 22)$, or $\mathrm{t}(15 ; 17)(\mathrm{q} 24 ; \mathrm{q} 21)-$ the patient is classified as being in a cytogenetically "favorable" risk group and is no longer "intermediate" and therefore, the gene expression markers are not applicable in this category. In this case, the results exclusively show "not applicable" on the report. AMLprofiler is designed to detect low expression levels of $B A A L C$ and high expression levels of $E V I 1$ genes. The expression marker results are a derivative of an evaluation of the measured expression level of the EVII or the $B A A L C$ marker against an expression threshold value, so called "cut-off point." EVI1 and BAALC marker results are currently validated for the "intermediate" cytogenetic risk group only. In case both BAALC and EVI1 show "detected," it is advised that EVI1 high "detected" should take precedence over the BAALC low "detected."

\section{Results}

53 adult patients diagnosed with de novo AML (based on a blast count of $>20 \%$ ) were recruited. We analysed 65 AML patient samples which included 49 from bone marrow and 16 from peripheral blood. Matching bone marrow and peripheral blood samples were collected from 12 patients. For this study, we recruited both male $(N=35)$ and female $(N=18)$ AML patients above 18 years of age. Our study included a combination of Caucasian and Black African AML patients from both the public and private health care sectors (Table 2). 
TABle 2: Sample characteristics.

\begin{tabular}{lc}
\hline Sample characteristic & Value \\
\hline Number of study participants & 53 \\
Number of samples analysed & 65 \\
Source of sample & \\
$\quad$ Bone marrow (BM) & 49 \\
$\quad$ Peripheral blood & 16 (with 12 matching BM) \\
Gender & $35(66 \%)$ \\
Male & $18(34 \%)$ \\
Female & \\
Race distribution & $20(37.7 \%)$ \\
$\quad$ Caucasian & $33(62.3 \%)$ \\
Black African & \\
Health sector & $35(66 \%)$ \\
Public & $18(34 \%)$ \\
Private & $44 \pm 17.4$ \\
Age distribution & $55 \% \pm 26 \%$ \\
Blast count &
\end{tabular}

Age distribution and blast count are expressed as mean \pm standard deviation.

When bone marrow samples were assessed, AMLprofiler detected 11 patients with chromosomal aberrations $(\mathfrak{t}(8 ; 21)$, $\operatorname{inv}(16) / t(16 ; 16)$, and/or $t(15 ; 17))$ and 22 patients with gene mutation and expression markers including NPM1mut, CEBPAdm, BAALC-low, and EVI1-high. 20 patients were found to be negative for all cytogenetic and molecular markers present on the AMLprofiler. 14 patients were found to be cytogenetically normal, and AMLprofiler allowed them to be categorized into favorable and poor risk groups. The group with a favorable prognosis included NPM1 $(N=2), B A A L C$ $(N=6), N P M 1+B A A L C(N=3)$, and CEBPA $(N=1)$. The categorization of the remaining two individuals into the poor prognosis group was based on increased levels of EVI1 expression. These two individuals also expressed low-level $B A A L C$, which is superseded in the analysis by increased EVI1 expression. Supplementary Table 1 available online at https://doi.org/10.1155/2017/2560191 provides all the results for individual patients.

With regard to the frequency of cytogenetic and molecular variables on the AMLprofiler, important differences were detected in some cases between the South African population and global averages (Table 3). These included a lower frequency of NPM1ABD variants in the South African group (9.4\%) when compared to the global average $(32.5 \%)$ and a higher frequency of increased EVI1 expression (18.9\%) when compared to the global average (9\%). We further detected a slightly lower frequency of CEBPA double mutants (1.9\%) in our cohort when compared to what has been reported globally (5-14\%).

In this study, the cost of standard modalities per patient ranged between USD 129 and USD 1167 (average USD 648). Although not offered as a service in South Africa, it is anticipated that the cost of an AMLprofiler diagnostic would be in the order of USD 1000. It is likely however that this would be reduced as economies of scale come to bear.
Turnaround time using standard methods was on average 20 days (ranging from 3 to 40 days) from sample collection. The AMLprofiler test can be executed in three days, and the final results can be provided in less than five days.

Our study also included the following results that were discordant: one of the patients was positive for the CEBPAdm mutations in the bone marrow sample, while this was negative in the corresponding peripheral blood sample. This indicates that the patient may have had a sporadic CEBPAassociated AML where the CEBPA mutations were acquired during the course of leukemogenesis [12]. One patient was found to have low expression of BAALC in the peripheral blood while being negative in the bone marrow, while another patient was positive for chromosomal aberration $t(15 ; 17)$ only in the bone marrow sample. These BM and PB discrepancies revealed by the AMLprofiler could not be compared to their corresponding routine test results as the latter were not requested in the course of the routine management of these patients. In addition, we detected both $t(8 ; 21)$, $\mathrm{t}(15 ; 17)$, and $\operatorname{inv}(16) / \mathrm{t}(16 ; 16)$ chromosomal aberrations with the AMLprofiler which are not requested in the standard modalities undertaken for AML patients in South Africa. However, our results suggest that AMLprofiler has the potential to change the prognostic stratification of AML patients who do not have major cytogenetic abnormalities, particularly since some of the tests are not currently requested or performed.

\section{Discussion}

In this study, we undertook a microarray-based assessment of molecular variables in AML including chromosomal aberrations, gene mutations, and alterations in gene expression, which offers high sensitivity and specificity and several advantages over diagnostic testing methods currently used. We also evaluated the cost factor and the turnaround time for performing diagnosis with both conventional methods and the AMLprofiler.

Although NPM1 mutations are the most frequent mutations detected in AML, occurring in $25 \%-30 \%$ of patients [13], in our study, the frequency of NPM1 mutations was low when compared to the global average. This suggests that the gene mutations assessed for on the microarray might be population specific and are therefore not being detected. In 2014, Marshall et al. reported a lower frequency of known NPM1 and FLT3-ITD mutations in a South African AML cohort and suggested that race-specific mutations might contribute to AML pathogenesis and also to the lower frequency of detection of these mutations [14]. They further concluded that NPM1 mutation frequency increases with population age and that their cohort had a considerably younger median age at diagnosis, which might contribute to the lower frequency of NPM1 mutations observed in their study. A similar age-dependent increase in the incidence of NPM1 mutations has previously been reported $[15,16]$. AMLprofiler does not investigate non-ABD mutations within the NPM1 gene, which indicates that other NPM1 mutations might be present in the South African population that could not be detected through this method. 
TABLE 3: Variant frequency of molecular markers on AMLproflier.

\begin{tabular}{lccccc}
\hline $\begin{array}{l}\text { Molecular markers on } \\
\text { AMLprofiler }\end{array}$ & $\begin{array}{c}\text { Frequency in our } \\
\text { current study }\end{array}$ & $\begin{array}{c}\text { Global frequency } \\
\text { in AML }\end{array}$ & $\begin{array}{c}\text { Frequency in Black } \\
\text { Africans }(n=33)\end{array}$ & $\begin{array}{c}\text { Frequency in } \\
\text { Caucasians }(n=20)\end{array}$ & References \\
\hline inv(16)(p13q22)/ & $5.7 \%$ & $5-10 \%$ & $1.9 \%$ & $3.8 \%$ & {$[5,21-27]$} \\
$\mathrm{t}(16 ; 16)(\mathrm{p} 13 ; \mathrm{q} 22)$ & $11.3 \%$ & $7 \%-10 \%$ & $5.6 \%$ & $5.7 \%$ & {$[5,26-30]$} \\
$\mathrm{t}(8 ; 21)(\mathrm{q} 22 ; \mathrm{q} 22)$ & $3.8 \%$ & $5-13 \%$ & $1.9 \%$ & $1.9 \%$ & {$[24,26,27,29$,} \\
$\mathrm{t}(15 ; 17)(\mathrm{q} 24 ; \mathrm{q} 21)$ & $1.9 \%$ & $5-14 \%$ & 0 & $1.9 \%$ & $31-33]$ \\
CEBPA double mutant & $9.4 \%$ & $25-40 \%$ & $1.9 \%$ & $7.5 \%$ & {$[13,16,41]$} \\
NPM1-ABD mutations & $18.9 \%$ & $8-10 \%$ & $15.1 \%$ & $3.8 \%$ & {$[10,17,48-50]$} \\
EVI1-high expression & $28.3 \%$ & $26 \%$ & $13.2 \%$ & $15.1 \%$ & {$[11]$} \\
BAALC-low expression & & & & & \\
\hline
\end{tabular}

AMLprofiler detects high levels of EVI1 expression in AML patients, which is a poor prognostic marker. Our results show a higher frequency of EVI1 gene expression compared to other studies that have reported high EVI1 expression [17]. Furthermore, AMLprofiler is designed to detect low expression of $B A A L C$, which is seen as a favorable prognostic marker. The majority of global studies have reported a high expression of BAALC, which is associated with an unfavorable prognosis $[9,18]$. However, in 2016, Nomdedéu et al. reported a $26 \%$ frequency of low BAALC expression in their AML cohort [11]. Therefore, only limited comparisons are possible with our $B A A L C$ results and only from a low expression point of view. The reason for the major difference in the variant numbers of NPM1 gene mutations between AMLprofiler and standard modalities in South Africa is likely to be due to the fact that the NPM1 mutation test is not routinely requested by clinicians in South Africa. In addition, neither $C E B P A$ nor $B A A L C$ tests are offered in the South African diagnostic setting.

Further investigations should be performed on larger cohorts of AML samples in the South African population to generate more information and to confirm our results using the AMLprofiler. Samples that are negative for molecular markers should undergo sequencing for the detection of other common biologically relevant mutations in AML including FLT3, KIT, RUNX1, DNMT3A, IDH1, TET2, and ASXL1. Next-generation sequencing (NGS) could possibly identify novel genetic markers in a population specific group and add further value to the results generated by AMLprofiler $[19,20]$. Further investigation of all the NPM1-negative cases using targeted sequencing could possibly identify non-ABD mutations that are common and specific to the South African population. Furthermore, incorporation of other molecular variables of prognostic value on the AMLprofiler might offer an advantage over the current version of the microarray.

Our data revealed that there are both advantages and limitations to using the AMLprofiler. First, AMLprofiler provides for a quicker genetic subtyping of AML and allows for better prognostic stratification of AML patients. An additional benefit is the comprehensive reporting with a quick turnaround time. While routine laboratory diagnostic test results usually take anywhere between 3 and 4 weeks and sometimes even longer, AMLprofiler significantly reduces time between sampling and diagnosis by providing results in 3 days and hence is much quicker in terms of reporting. Since AML progresses rapidly, diagnosis and prognosis play a crucial role in the treatment of these patients. AMLprofiler allows quicker diagnosis as well as prognostic stratification which translates into earlier treatment decisions and also provides valuable time for finding suitable stem cell donors, if applicable. Hence, AMLprofiler enables the clinician to provide the right treatment for AML patients especially in cases which fall into the intermediate risk group with no major chromosomal aberrations.

Furthermore, due to the full standardization of the test, standardized and comparable results can be generated at any diagnostic lab. The costs of implementing and running an AMLprofiler test are also significantly lower when compared to the costs of developing and validating an in-house set of tests in each diagnostic lab. Routine clinical diagnostic methods for AML in South Africa involve cytogenetic testing, FISH, karyotyping, and PCR for detection of chromosomal aberrations and molecular variants which often are time-consuming and tedious laboratory procedures. AMLprofiler replaces seven separate assays including 3 chromosomal aberrations, 2 gene mutations, and 2 gene expression changes in one single test, which saves a considerable amount of time and labor. Hence, AMLprofiler covers a wide range of molecular markers that are used for standard AML diagnosis since it presents a single platform for detection of seven diagnostic markers. AMLprofiler has an added benefit in terms of high specificity and sensitivity with gene expression markers as it is designed to detect low levels of BAALC expression and high levels of EVI1 expression.

Notwithstanding the benefits provided by the AMLprofiler, the absence on this microarray of mutations in the FLT3 gene, which is a crucial prognostic marker for AML, is a significant limitation. Therefore, a fully comprehensive diagnosis will require a separate investigation of FLT3 variants. In addition, the AMLprofiler does not report non-ABD mutations in the NPM1 gene. Finally, the AMLprofiler requires a sophisticated platform for processing that is not common in diagnostic laboratories in South Africa. 


\section{Conclusion}

Although microarray technology is not being utilized routinely for AML diagnosis in the South African clinical setting, it might prove to be an important tool to categorize AML patients into prognostic risk groups and thereby assist clinicians to provide tailored therapy for these patients. AMLprofiler offers a more comprehensive investigation of AML samples, adds significant prognostic value, and decreases time to result. AMLprofiler also offers consistent pricing when compared to standard modalities in the current setting in South Africa, and this is likely to be reduced when economies of scale come to bear. We therefore conclude that the AMLproflier would be a useful tool for AML diagnosis and patient stratification in the South African clinical setting.

\section{Conflicts of Interest}

The authors declare that they have no conflicts of interest.

\section{Acknowledgments}

The authors would like to sincerely thank SkylineDx, Rotterdam, The Netherlands, for the provision of AMLprofilers and their continuous assistance. The authors also thank the Department of Haematology and NHLS partners at the University of Pretoria, Ampath, Vermaak and Vennote, Dr. Albert's Cellular Therapy, and Dr. Brittain and partners for providing AML samples. This work was funded by the University of Pretoria (Institute for Cellular and Molecular Medicine and Vice Chancellor's post-doctoral fellowship), the National Health Laboratory Services Research Trust, the South African Medical Research Council (Category 1 University Flagship and Extramural Stem Cell Unit grants), and the National Research Foundation of South Africa.

\section{References}

[1] A. Walker and G. Marcucci, "Molecular prognostic factors in cytogenetically normal acute myeloid leukemia," Expert Review of Hematology, vol. 5, no. 5, pp. 547-558, 2012.

[2] S. J. McKenna, "Leukemia," Oral Surgery, Oral Medicine, Oral Pathology, Oral Radiology, and Endodontics, vol. 89, no. 2, pp. 137-139, 2000.

[3] H. Döhner, E. H. Estey, S. Amadori et al., "Diagnosis and management of acute myeloid leukemia in adults: recommendations from an international expert panel, on behalf of the European LeukemiaNet," Blood, vol. 115, no. 3, pp. 453-474, 2010.

[4] J. W. Vardiman, J. Thiele, D. A. Arber et al., "The 2008 revision of the World Health Organization (WHO) classification of myeloid neoplasms and acute leukemia: rationale and important changes," Blood, vol. 114, no. 5, pp. 937-951, 2009.

[5] D. Grimwade, R. K. Hills, A. V. Moorman et al., "Refinement of cytogenetic classification in acute myeloid leukemia: determination of prognostic significance of rare recurring chromosomal abnormalities among 5876 younger adult patients treated in the United Kingdom Medical Research Council trials," Blood, vol. 116, no. 3, pp. 354-365, 2010.

[6] R. S. Ohgami, L. Ma, J. D. Merker et al., "Next-generation sequencing of acute myeloid leukemia identifies the significance of TP53, U2AF1, ASXL1, and TET2 mutations," Modern Pathology, vol. 28, no. 5, pp. 706-714, 2015.

[7] H. Döhner, E. Estey, D. Grimwade et al., "Diagnosis and management of AML in adults: 2017 ELN recommendations from an international expert panel," Blood, vol. 129, no. 4, pp. 424447, 2017.

[8] S. Schwind, G. Marcucci, J. Kohlschmidt et al., "Low expression of MN1 associates with better treatment response in older patients with de novo cytogenetically normal acute myeloid leukemia," Blood, vol. 118, no. 15, pp. 4188-4198, 2011.

[9] C. Langer, M. D. Radmacher, A. S. Ruppert et al., "High $B A A L C$ expression associates with other molecular prognostic markers, poor outcome, and a distinct gene-expression signature in cytogenetically normal patients younger than 60 years with acute myeloid leukemia: a Cancer and Leukemia Group B (CALGB) study," Blood, vol. 111, no. 11, pp. 5371-5379, 2008.

[10] J. Brand, M. H. van Vliet, L. de Best et al., “A standardized microarray assay for the independent gene expression markers in AML: EVI1 and BAALC," Experimental Hematology of Oncology, vol. 2, no. 1, p. 7, 2013.

[11] J. F. Nomdedéu, E. Puigdecanet, E. Bussaglia et al., "Feasibility of the AML profiler (Skyline ${ }^{\mathrm{TM}}$ Array) for patient risk stratification in a multicentre trial: a preliminary comparison with the conventional approach," Hematological Oncology, 2016.

[12] K. Tawana, A. Rio-Machin, C. Preudhomme, and J. Fitzgibbon, "Familial CEBPA-mutated acute myeloid leukemia," Seminars in Hematology, vol. 54, no. 2, pp. 87-93, 2017.

[13] S. Schnittger, C. Schoch, W. Kern et al., "Nucleophosmin gene mutations are predictors of favorable prognosis in acute myelogenous leukemia with a normal karyotype," Blood, vol. 106, no. 12, pp. 3733-3739, 2005.

[14] R. C. Marshall, A. Tlagadi, M. Bronze et al., "Lower frequency of NPM1 and FLT3-ITD mutations in a South African adult de novo AML cohort," International Journal of Laboratory Hematology, vol. 36, no. 6, pp. 656-664, 2014.

[15] W. C. Chou, J. L. Tang, L. I. Lin et al., "Nucleophosmin mutations in de novo acute myeloid leukemia: the age-dependent incidences and the stability during disease evolution," Cancer Research, vol. 66, no. 6, pp. 3310-3316, 2006.

[16] F. Ahmad, S. Mandava, and B. R. Das, "Mutations of NPM1 gene in de novo acute myeloid leukaemia: determination of incidence, distribution pattern and identification of two novel mutations in Indian population," Hematological Oncology, vol. 27, no. 2, pp. 90-97, 2009.

[17] S. Lugthart, E. van Drunen, Y. van Norden et al., "High EVI1 levels predict adverse outcome in acute myeloid leukemia: prevalence of EVI1 overexpression and chromosome $3 \mathrm{q} 26$ abnormalities underestimated," Blood, vol. 111, no. 8, pp. 4329-4337, 2008.

[18] S. J. Xiao, J. Z. Shen, J. L. Huang, and H. Y. Fu, "Prognostic significance of the BAALC gene expression in adult patients with acute myeloid leukemia: a meta-analysis," Molecular and Clinical Oncology, vol. 3, no. 4, pp. 880-888, 2015.

[19] A. M. Ilyas, S. Ahmad, M. Faheem et al., "Next generation sequencing of acute myeloid leukemia: influencing prognosis," BMC Genomics, vol. 16, Supplement 1, p. S5, 2015.

[20] R. Luthra, K. P. Patel, N. G. Reddy et al., "Next-generation sequencing-based multigene mutational screening for acute myeloid leukemia using Miseq: applicability for diagnostics 
and disease monitoring," Haematologica, vol. 99, no. 3, pp. 465-473, 2014.

[21] J. Delaunay, N. Vey, T. Leblanc et al., "Prognosis of inv(16)/ $t(16 ; 16)$ acute myeloid leukemia (AML): a survey of 110 cases from the French AML Intergroup," Blood, vol. 102, no. 2, pp. 462-469, 2003.

[22] R. Billstrom, T. Ahlgren, A. N. Bekassy et al., “Acute myeloid leukemia with inv(16)(p13q22): involvement of cervical lymph nodes and tonsils is common and may be a negative prognostic sign," American Journal of Hematology, vol. 71, no. 1, pp. 15$19,2002$.

[23] K. Mrozek, N. A. Heerema, and C. D. Bloomfield, "Cytogenetics in acute leukemia," Blood Reviews, vol. 18, no. 2, pp. 115136, 2004.

[24] J. C. Byrd, K. Mrózek, R. K. Dodge et al., "Pretreatment cytogenetic abnormalities are predictive of induction success, cumulative incidence of relapse, and overall survival in adult patients with de novo acute myeloid leukemia: results from Cancer and Leukemia Group B (CALGB 8461)," Blood, vol. 100, no. 13, pp. 4325-4336, 2002.

[25] S. Schwind, C. G. Edwards, D. Nicolet et al., "inv(16)/t(16;16) acute myeloid leukemia with non-type A CBFB-MYH11 fusions associate with distinct clinical and genetic features and lack KIT mutations," Blood, vol. 121, no. 2, pp. 385-391, 2013.

[26] K. Mrozek and C. D. Bloomfield, "Clinical significance of the most common chromosome translocations in adult acute myeloid leukemia," Journal of the National Cancer Institute Monographs, vol. 2008, no. 39, pp. 52-57, 2008.

[27] D. Grimwade, "The clinical significance of cytogenetic abnormalities in acute myeloid leukaemia," Best Practice \& Research Clinical Haematology, vol. 14, no. 3, pp. 497-529, 2001.

[28] P. J. Valk, R. G. Verhaak, M. A. Beijen et al., "Prognostically useful gene-expression profiles in acute myeloid leukemia," The New England Journal of Medicine, vol. 350, no. 16, pp. 1617-1628, 2004.

[29] Y. Cheng, Y. Wang, H. Wang et al., "Cytogenetic profile of de novo acute myeloid leukemia: a study based on 1432 patients in a single institution of China," Leukemia, vol. 23, no. 10, pp. 1801-1806, 2009.

[30] D. A. Arber, A. S. Stein, N. H. Carter, D. Ikle, S. J. Forman, and M. L. Slovak, "Prognostic impact of acute myeloid leukemia classification: importance of detection of recurring cytogenetic abnormalities and multilineage dysplasia on survival," American Journal of Clinical Pathology, vol. 119, no. 5, pp. 672-680, 2003.

[31] M. L. Chauffaille, M. S. Figueiredo, R. Beltrani, S. V. Antunes, M. Yamamoto, and J. Kerbauy, "Acute promyelocytic leukemia: the study of $\mathrm{t}(15 ; 17)$ translocation by fluorescent in situ hybridization, reverse transcriptase-polymerase chain reaction and cytogenetic techniques," Brazilian Journal of Medical and Biological Research, vol. 34, no. 6, pp. 735-743, 2001.

[32] K. Mrozek, "Cytogenetic, molecular genetic, and clinical characteristics of acute myeloid leukemia with a complex karyotype," Seminars in Oncology, vol. 35, no. 4, pp. 365377, 2008.

[33] C. Y. Meng, P. J. Noor, A. Ismail, M. F. Ahid, and Z. Zakaria, "Cytogenetic profile of de novo acute myeloid leukemia patients in Malaysia," International Journal of Biomedical Sciences, vol. 9, no. 1, pp. 26-32, 2013.
[34] T. Pabst, B. U. Mueller, P. Zhang et al., "Dominant-negative mutations of CEBPA, encoding CCAAT/enhancer binding protein- $\alpha(\mathrm{C} / \mathrm{EBP} \alpha)$, in acute myeloid leukemia," Nature Genetics, vol. 27, no. 3, pp. 263-270, 2001.

[35] A. F. Gombart, W. K. Hofmann, S. Kawano et al., "Mutations in the gene encoding the transcription factor CCAAT/ enhancer binding protein $\alpha$ in myelodysplastic syndromes and acute myeloid leukemias," Blood, vol. 99, no. 4, pp. 1332-1340, 2002.

[36] C. Preudhomme, C. Sagot, N. Boissel et al., "Favorable prognostic significance of CEBPA mutations in patients with de novo acute myeloid leukemia: a study from the acute leukemia French association (ALFA)," Blood, vol. 100, no. 8, pp. $2717-$ 2723, 2002.

[37] J. Snaddon, M. L. Smith, M. Neat et al., "Mutations of CEBPA in acute myeloid leukemia FAB types M1 and M2," Genes, Chromosomes and Cancer, vol. 37, no. 1, pp. 72-78, 2003.

[38] S. Barjesteh van Waalwijk van Doorn-Khosrovani, C. Erpelinck, J. Meijer et al., "Biallelic mutations in the CEBPA gene and low CEBPA expression levels as prognostic markers in intermediate-risk AML," The Hematology Journal, vol. 4, no. 1, pp. 31-40, 2003.

[39] S. Fröhling and H. Döhner, "Disruption of C/EBP $\alpha$ function in acute myeloid leukemia," The New England Journal of Medicine, vol. 351, no. 23, pp. 2370-2372, 2004.

[40] C. Nerlov, "C/EBP $\alpha$ mutations in acute myeloid leukaemias," Nature Reviews Cancer, vol. 4, no. 5, pp. 394-400, 2004.

[41] M. Bienz, M. Ludwig, E. O. Leibundgut et al., "Risk assessment in patients with acute myeloid leukemia and a normal karyotype," Clinical Cancer Research, vol. 11, no. 4, pp. 1416-1424, 2005.

[42] B. Falini, I. Nicoletti, M. F. Martelli, and C. Mecucci, "Acute myeloid leukemia carrying cytoplasmic/mutated nucleophosmin $\left(\mathrm{NPMc}^{+} \mathrm{AML}\right)$ : biologic and clinical features," Blood, vol. 109, no. 3, pp. 874-885, 2007.

[43] C. Thiede, S. Koch, E. Creutzig et al., "Prevalence and prognostic impact of NPM1 mutations in 1485 adult patients with acute myeloid leukemia (AML)," Blood, vol. 107, no. 10, pp. 4011-4020, 2006.

[44] G. Cazzaniga, M. G. Dell'Oro, C. Mecucci et al., "Nucleophosmin mutations in childhood acute myelogenous leukemia with normal karyotype," Blood, vol. 106, no. 4, pp. 1419-1422, 2005.

[45] R. G. Verhaak, C. S. Goudswaard, W. van Putten et al., "Mutations in nucleophosmin (NPM1) in acute myeloid leukemia (AML): association with other gene abnormalities and previously established gene expression signatures and their favorable prognostic significance," Blood, vol. 106, no. 12, pp. 3747-3754, 2005.

[46] P. Brown, E. McIntyre, R. Rau et al., "The incidence and clinical significance of nucleophosmin mutations in childhood AML," Blood, vol. 110, no. 3, pp. 979-985, 2007.

[47] G. R. Ruan, J. L. Li, Y. Z. Qin et al., "Nucleophosmin mutations in Chinese adults with acute myelogenous leukemia," Annals of Hematology, vol. 88, no. 2, pp. 159-166, 2009.

[48] S. Gröschel, S. Lugthart, R. F. Schlenk et al., "High EVI1 expression predicts outcome in younger adult patients with acute myeloid leukemia and is associated with distinct cytogenetic abnormalities," Journal of Clinical Oncology, vol. 28, no. 12, pp. 2101-2107, 2010. 
[49] C. Glass, M. Wilson, R. Gonzalez, Y. Zhang, and A. S. Perkins, "The role of EVI1 in myeloid malignancies," Blood Cells, Molecules \& Diseases, vol. 53, no. 1-2, pp. 67-76, 2014.

[50] S. Barjesteh van Waalwijk van Doorn-Khosrovani, C. Erpelinck, W. L. van Putten et al., "High EVI1 expression predicts poor survival in acute myeloid leukemia: a study of 319 de novo AML patients," Blood, vol. 101, no. 3, pp. 837$845,2003$. 

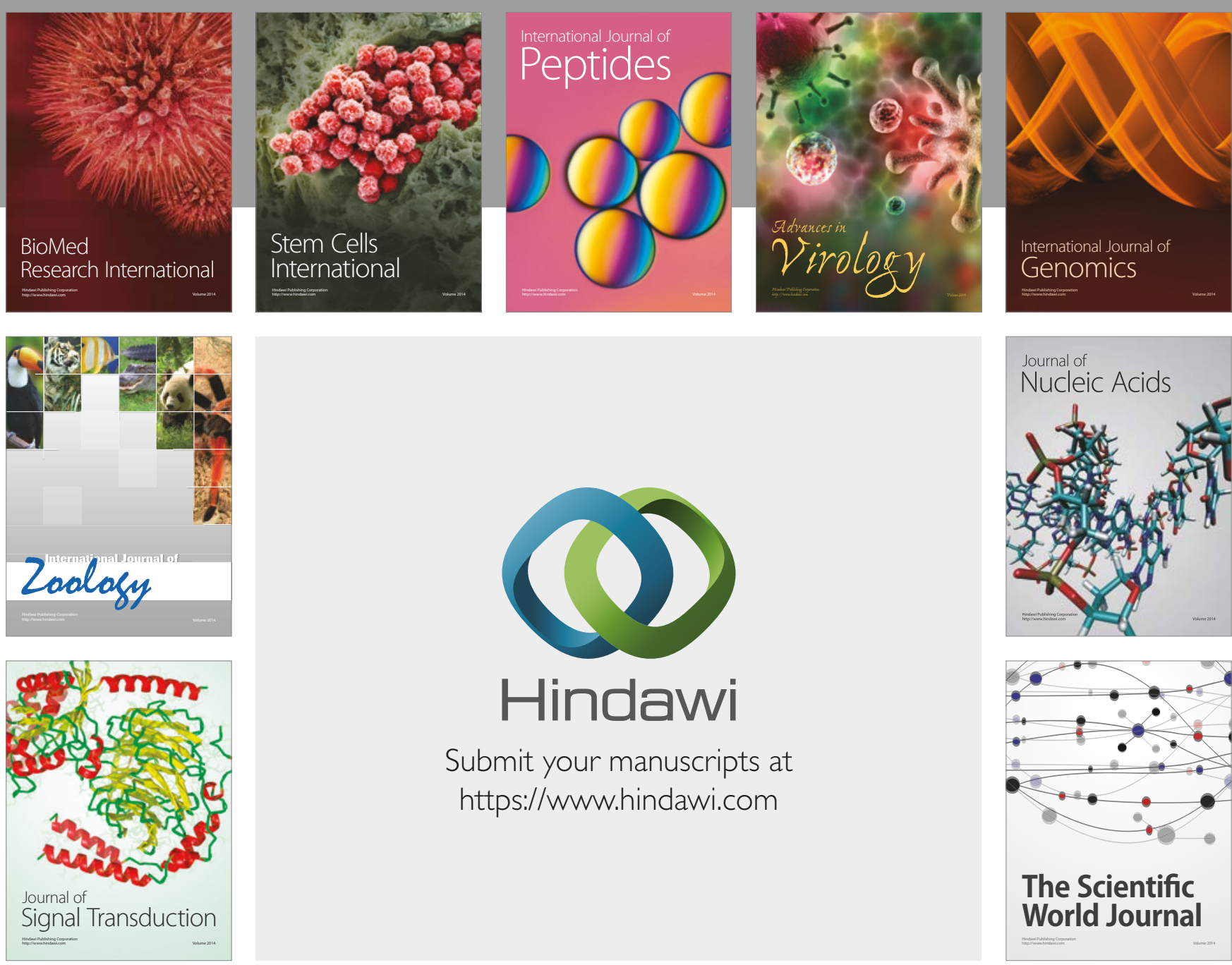

Submit your manuscripts at

https://www.hindawi.com
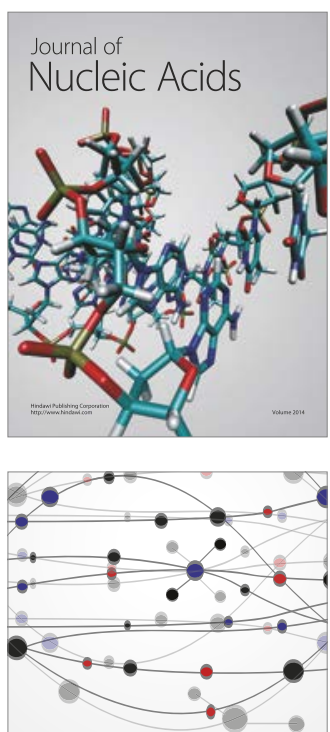

The Scientific World Journal

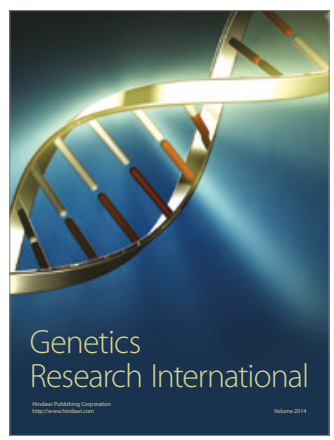

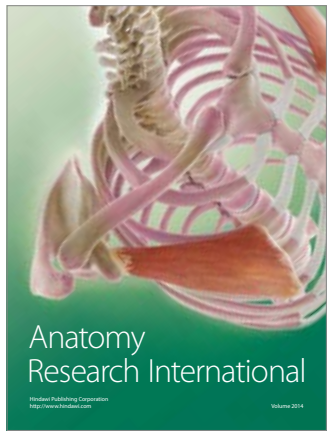

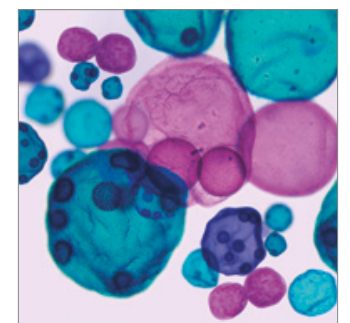

International Journal of Microbiology
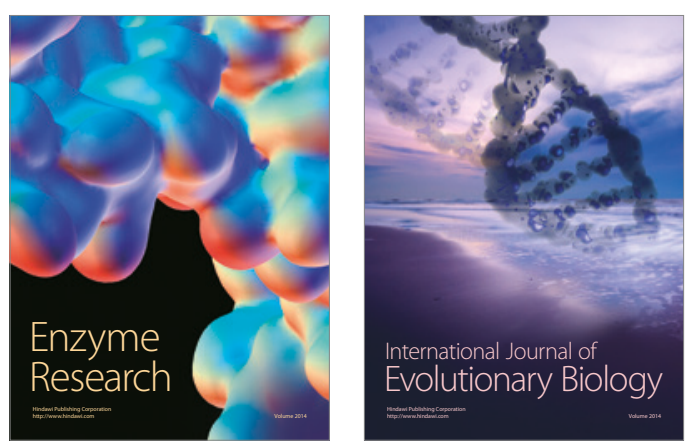
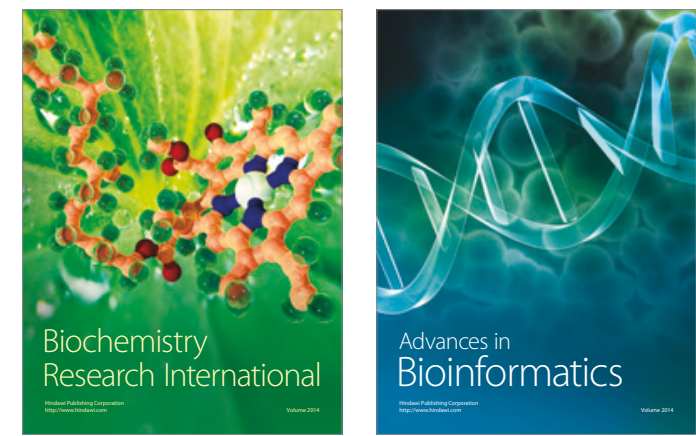

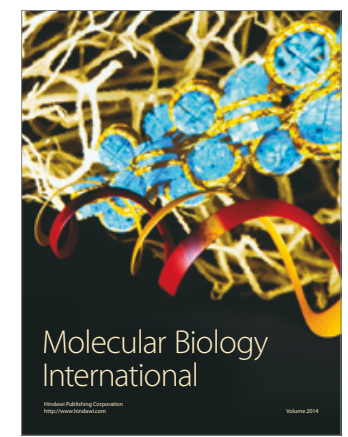

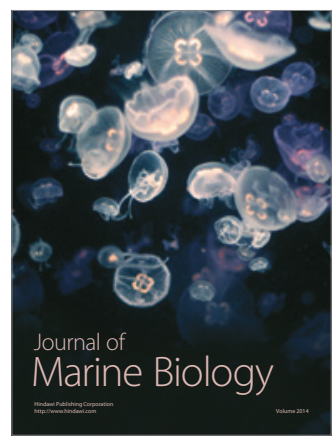

